\title{
Presentación
}

\section{Estrategias de formación de intelectuales y políticos de la educación'}

Este número se enmarca en el contexto de un proyecto de investigación que inspiró seminarios de posgrado y generó nuevos abordajes e investigaciones que se publican en esta edición de la revista. Todo comienza con una investigación sobre Circulación Internacional y Formación de Cuadros Dirigentes, desarrollada en la Universidad Estadual de Campinas (2006/2011)2. Se trata de una investigación cuyo aporte teórico viene de Bourdieu, Dezalay, Karady, Cooper. La propia línea de estudios fue formulada por Pierre Bourdieu y desarrollada por Dezalay y Garth (BOURDIEU, 1981; DEZALAY, 2002, 2004; KARADY, 1998; COOPER, 2001).

Dentro de este proyecto, al estudiar la trayectoria del intelectual Anísio Teixeira examiné los efectos de sus viajes internacionales llevados a cabo como parte de su formación. Busqué relacionar, en la trayectoria de uno de los primeros educadores profesionales brasileños, el impacto de la formación cosmopolita en los proyectos que puso en marcha, especialmente en lo relativo a la creación del sistema nacional de educación en Brasil ${ }^{3}$. Resultados de esa investigación fueron transformados en las indagaciones que nortearon los dos seminarios realizados: en La Facultad Latinoamericana de Ciencias Sociales - FLACSO, en Buenos Aires y en la Universidad Pedagógica Nacional, en Bogotá en el año 2010. Los seminarios inspiraron nuevas miradas por parte de los estudiantes en sus investigaciones en curso. Se ampliaron

1 Agradezco a la profesora Martha Cecilia Herrera sin cuyo apoyo este número no habría sido posible. Agradezco especialmente su invitación como profesora visitante y la acogida en su grupo de investigación: Educación y Cultura Política durante este período, en el segundo semestre de 2010, al Doctorado Interinstitucional en Educación en la sede UPN, así como sus sugerencias y críticas a la organización de este número de la revista y la traducción de esta presentación.

2 Circulación Internacional y Formación de Cuadros Dirigentes, proyecto coordinado por la profesora Letícia Bicalho Canedo y financiado por la FAPESP (Brasil).

3 Algunas publicaciones de este proyecto pueden ser consultadas en: Almeida, A. M. Canêdo, L.B., Garcia, A. Bittencourt, A. B., Circulação Internacional e Formação Intelectual das Elites Brasileiras. Campinas, SP: Editora da Unicamp, 2004. Herrera, M. C.; Bittencourt, A. B. Política, Intelectuales y espacio publico en las sociedades contemporáneas, Bogotá: Cooperativa Editorial Magisterio, 2007. Mobilité universitaire et circulation internationale des idées: le Brésil et la mondialisation des savoirs (Cahiers de la recherche sur l'éducation et les savoirs, hors-série n²/juin 2009). 
sus objetos de investigación al mismo tiempo que se profundizaron sus propios interrogantes.

La organización de este número de la Revista Colombiana de Educación tiene por base los resultados de esas investigaciones a los cuales se sumaron otros investigadores invitados por una convocatoria pública de la revista hecha a finales de 2010.

La circulación internacional es un problema que ha interesado a muchos pero no es un problema nuevo, fue parte importante de la formación de las elites intelectuales no solo en América Latina, cuya historia colonial imponía la circulación entre la metrópoli y la colonia, sino también en la misma Europa, como nos indican las biografías de intelectuales, artistas y escritores, o como comprueba Viktor Karady en su estudio sobre la circulación de los estudiantes europeos, en el siglo XIX (KARADY, 1998). Tal desplazamiento de personas opera un movimiento de ideas, objetivado a través de la circulación de textos escritos importados y traducidos, que impacta el desarrollo del pensamiento de comunidades intelectuales en distintos espacios. Pierre Bourdieu, al llamar la atención de los cientistas sociales sobre sus responsabilidades como productores de discursos legítimos, analizó los riesgos de la importación de ideas y teorías sin considerar las condiciones de su producción original (BOURDIEU, 1981). En este sentido, él mismo y los autores que le siguieran hacen el ejercicio de análisis en torno al modo como ideas y teorías adecuadas a una dada realidad, al ser importadas sin su contexto, pueden transformarse en instrumento de dominación y alienación. Se convierten de instrumento para la solución de un problema históricamente localizado, en elementos de doctrina con pretensiones de universalidad.

La importación/exportación de ideas y de intelectuales no se presenta de manera espontánea. Se trata de un proceso que involucra los intereses individuales y de ciertos grupos, al tiempo que forma parte de las políticas de Estado. La modernidad se construyó bajo la hegemonía europea especialmente de Francia, Inglaterra, España y Portugal. Las dos guerras mundiales cambiaron el panorama internacional en el siglo $\mathrm{XX}$ y sustituyeron el centro hegemónico europeo por el de los Estados Unidos de América.

El proceso de construcción de la nueva hegemonía no se dio por arte de magia, a pesar de que las dos guerras mundiales constituyan el marco de esa nueva orientación internacional, fue necesario un largo camino de elaboración de la legitimidad americana para poder ejercer la conducción de la política internacional. Esta alteración del centro hegemónico no significó simplemente una alteración en la geopolítica sino también una alteración del mismo paradigma de gobernanza. Y en ese proceso los intelectuales, las universidades, los gobiernos, los grandes aparatos políticos como iglesias, sin- 
dicatos y organizaciones de diverso tipo jugaron papel relevante. Yves Dezalay examina la construcción del nuevo paradigma político que se volvería dominante después de la segunda guerra mundial, señalando como todo el proceso de sustitución de los abogados por los economistas, conocidos como los estrategas de los Estados contemporáneos, fue desarrollado en universidades norteamericanas y difundido gracias a los esfuerzos de los gobiernos, de las fundaciones filantrópicas, de los organismos internacionales (ONU, UNESCO, BID, Banco Mundial) y de las propias políticas de internacionalización de las grandes universidades americanas (DEZALAY, 2004; DEZALAY \& GARTH, 2002). En La mondialisation des guerres de palais los autores tratan de la preparación de los economistas que conformaron el equipo de los gobiernos de Brasil, Chile, México y Argentina y posibilitaron las reformas de Estado en los cuatro países, durante las décadas de 1970 y 1980, bajo la égida norteamericana. La tesis de Dezalay tiene como punto de partida la hipótesis formulada por Bourdieu sobre los efectos problemáticos de la importación de teorías sin sus contextos de producción y se inserta en los debates sobre la mundialización de la economía y la globalización de los procesos políticos.

Las referencias al proceso reciente de globalización, difundidas a partir de la década del 1990, basadas en la idea de que el mundo se transformaba teniendo como característica la desaparición de las fronteras nacionales, fueron acogidas por intelectuales de distintas líneas ideológicas, tomando la circulación internacional como un proceso generalizado y como un fenómeno totalmente nuevo. Este discurso está siendo todavía debatido por investigadores que estudian procesos históricos en su larga duración o la historia de formación de las elites nacionales.

Frederick Cooper, historiador del proceso de descolonización de África, describe la creación y continuidad de redes internacionales de cooperación entre grupos de interés de los países coloniales y de los colonizados, a través de las cuales la explotación se ejerce sin distinción entre los colonizadores y las elites autóctonas. Sus conclusiones más significativas son extraídas de los datos estadísticos que confirman sus hipótesis sobre la globalización no como un fenómeno nuevo sino que es antiguo, las elites siempre han Estado globalizadas. Comprueba con sus datos que la circulación de personas entre naciones fue mucho mayor en el fin del siglo XIX y en el inicio del siglo XX que en la década 
de 1990. La colonización y la esclavitud ya movilizaron contingentes humanos muy expresivos algo comparable al reciente proceso de globalización. De sus estudios surge la tesis de que existe permanencia en las redes que han sido establecidas por un proyecto político específico y que son activadas en otros momentos por proyectos muy diferentes de aquellos para los cuales éstas fueron conformadas originalmente.

Sus críticas a los conceptos de mundialización y globalización indican el uso político de los conceptos. Para Cooper fue el interés de los grupos dominantes en transformar la internacionalización del capital en un proceso de internacionalización general el que activó la difusión de estos conceptos, proceso potencializado por grupos de izquierda que, en el afán de criticar el avance de grupos económicos multinacionales sobre las economías periféricas, contribuyeron a afirmar conceptos que no expresan la realidad histórica - la Fanfaronnade du banquier / la Lamentation social-démocrate (COOPER, 2001, p. 103/104). En este debate sobre la mundialización se pueden identificar los intereses de los Estados centrales en controlar la circulación de poblaciones pobres para no sobrecargar sus economías, mantenidas gracias a la explotación de las sociedades periféricas valiéndose, especialmente, del frágil sistema de protección al trabajo en estas últimas. Al tiempo que se mantienen las puertas abiertas a los contingen- tes de estudiantes y de técnicos bien formados y provenientes de los mismos países periféricos, ocasionando la llamada fuga de cerebros de los países pobres. Los dramas de los inmigrantes ilegales y el aumento en el número de becas de estudio se configuran en ejemplos de esa circulación deseada o reprimida.

La dirección de la circulación más deseada parece ser semejante a la de la más reprimida e indica el lugar en el que están situados los centros hegemónicos. Desde la posguerra, las elites latinoamericanas dirigieron su mirada hacia los Estados Unidos, al igual que grandes contingentes de pobres y de desempleados que siguieron la misma dirección con destino diferente. No solo se importó teoría y tecnología, el mismo proceso de industrialización en los moldes norteamericanos exigió también de la formación de técnicos, científicos e ingenieros por encima de la organización de un sistema educativo pragmático. De este modo, las grandes naciones latinoamericanas, como Brasil, México, Chile, Colombia, Argentina, fueron impactadas por reformas importadas en las décadas del sesenta y setenta. Y en los años noventa nuevas reformas del Estado se propagan por el continente. Estas reformas fueran posibilitadas por los mismos intelectuales que fueron formados en las sedes exportadoras de las propuestas de reformas (CORBALÁN, 2002).

Los artículos que vamos a leer en este número de la Revista Colombiana de Educación están organizados en dos 
partes: en la primera se estudian las trayectorias de técnicos, intelectuales y políticos latinoamericanos que fueron marcados por la circulación internacional y que protagonizaron reformas e innovaciones en la educación y en la cultura en sus respectivos países. En la segunda parte se agrupan trayectorias de profesores y sus redes sociales, cuyo trabajo logró crear nuevas disciplinas académicas, implementar nuevas áreas y prácticas en sus campos profesionales. Son maestros que alcanzaron el reconocimiento de sus pares por el impacto político de su trayectoria académica.

\section{Parte l: políticos, intelectuales y reformadores}

El examen de las trayectorias reunidas en la primera parte de este número permite comprender las estrategias usadas por los distintos agentes sociales para la acumulación y movilización de sus capitales en la construcción de carreras intelectuales notables y que los hicieron situar en el centro del escenario político nacional en sus respectivos países. En este conjunto de estudios, sobre personajes oriundos de distintos países de América Latina, emergen las organizaciones, las agencias y los mecanismos responsables por la formación de las elites nacionales. La Iglesia Católica, especialmente por sus colegios jesuitas, sus universidades y sus organizaciones para laicos, marca la formación de cuadros políticos especialmente en Colombia y en Brasil, como se puede ver en los artículos sobre Fernán González de Diego H. Arias, Gonzalo Sánchez de José Gabriel Cristancho, Carlos Eduardo Vasco de Milton Molano Camargo, Anísio Teixeira de Agueda Bernardete Bittencourt, y Carolina Ribeiro de Ana Regina Pinheiro. En todos estos casos el paso por las organizaciones católicas configuró la formación de redes de solidaridad que se mostraron imprescindibles en el establecimiento de las carreras profesionales y en las prácticas políticas de estos agentes.

Fundaciones filantrópicas internacionales como la Fundación Ford, Fundación Rockefeller con sus becas de estudio, especialmente hasta los años 60, así como las grandes universidades americanas con política de acogimiento de estudiantes extranjeros, como la Columbia University, Harvard, Berkeley, configuran un segundo espacio común en la formación de esos líderes en América Latina, como demuestran de nuevo los artículos de Agueda Bittencourt, Milton Molano Camargo además del de Andrés Klaus Runge y Diego Alejandro Muñoz sobre Luis López de Mesa. 
Las generaciones posteriores cuentan con nuevos agentes implicados en la formación de técnicos y dirigentes para los Estados nacionales: los organismos internacionales emergentes después de la Segunda Guerra como la UNESCO, el Banco Mundial, el Banco Interamericano de Desarrollo, la Comisión Económica para América Latina y el Caribe CEPAL, la FLACSO (Facultad Latinoamericana de Ciencias Sociales) y el ILPES (Instituto Latinoamericano de Planificación Económica y Social) que entran en el escenario político en América Latina. El estudio de Pablo Domínguez Vaselli sobre Germán W. Rama y el de Claudio Suasnábar y Nicolás Isola sobre la trayectoria de Tomás Amadeo Vasconi, ilustran el proceso de formación de importantes cuadros responsables de dirigir la política en la región. Trayectorias semejantes tuvieron los políticos brasileños: Fernando Henrique Cardoso, así como su ministro de educación Paulo Renato Souza y José Serra, contemporáneos de los otros intelectuales incluidos en este número ${ }^{4}$. Este conjunto de estudios permite percibir aún que la mayoría de los intelectuales políticos con larga permanencia en el poder en los Estados nacionales se concentra mayor cantidad de capitales: a los capitales familiares (económicos y sociales), se suman los del campo

4 Sobre este tema se puede consultar el trabajo de Fernanda Beigel: "La Flacso chilena y la regionalización de las ciencias sociales en América Latina (1957-1973)", Revista Mexicana de Sociología 71 (2): 319-349. religioso (redes de solidaridad), así como los conocimientos linguísticos; en general manejan varios idiomas y disfrutan de una cultura cosmopolita adquirida en viajes por Europa (Francia, Alemania, Italia) y Estados Unidos. Son ejemplo de esto las trayectorias de Carlos Eduardo Vasco Uribe y Anísio Teixeira.

En las últimas décadas la circulación internacional de estudiantes e intelectuales pasó a ser fomentada y controlada por los Estados. El estudio que cierra esta primera parte presenta la estructuración y las disputas de grupos en la constitución de un organismo público de fomento a la investigación, especialmente dedicado a la formación de personal de nivel superior, a través de invitaciones de misiones extranjeras y el otorgamiento de becas de estudio en el país y en el extranjero. La Comisión de Perfeccionamiento de Personal de Nivel Superior - CAPES al lado del Consejo Nacional de Investigación - CNPq fundados en los años 1950/1951, aparece como una de las primeras agencias del género en la región. Posteriormente (1958) fue creado el Consejo Nacional de Investigaciones Científicas y Técnicas - CONICET en Argentina y, en 1968, aparece Colciencias y el Consejo Nacional de Investigaciones Científicas y Técnicas, de Colombia. Estos nuevos organismos públicos imprimen las marcas de las políticas nacionales para la formación de los agentes ligados a la educación, la ciencia y la tecnología en la región. 


\section{Parte II: Intelectuales y Profesores}

En la segunda parte se reúnen los resultados de trabajos sobre las trayectorias de profesores que pueden ser sintetizados por la afirmación de Rocío Pérez Mesa y Guillermo Fonseca respecto a Eloísa Vasco Montoya, quien a lo largo de su vida aportó en la configuración de un nuevo maestro, desde su reconocimiento como productor de saber pedagógico, categoría que es vigente en el debate contemporáneo respecto a la pedagogía y la formación de profesores. Eloísa Vasco, cuya formación estuvo marcada por la Iglesia Católica y por repetidos viajes al exterior.

En el texto de Aydêe Luisa Robayo sobre José Agustín Blanco, la autora intenta mantener el formato de entrevista donde se entrelazan las historias del entrevistado con las interpretaciones de la propia autora en una construcción textual original y creativa. José Agustín Blanco fue el responsable por la implantación de la geografía y de la cartografía moderna en Colombia y llegó a ser una referencia internacional en su especialidad.

Fueron incluidos también en esta segunda parte otras trayectorias cuyos procesos de circulación internacional estuvieron garantizados por factores particulares. La de Gabriela Mistral en la lectura de Carola Sepúlveda, cuyas relaciones construidas, en el interior de Chile, a comienzos de su carrera de profesora, fueron la base para su posterior salida del país y su proyección internacional como poetisa, lo cual repercutió en la consecución del primer Premio Nobel de Literatura en América Latina. Miguel Fornaguera, el catalán exilado durante la Guerra Civil española mantuvo su red de solidaridad hasta finales de su vida, afirmando sus principios, defendiendo la libertad de los pueblos. Tuvo su formación marcada por un viaje de estudios a Paris, entonces centro cultural y académico, de paso obligatorio para los intelectuales de la primera mitad del siglo XX. Los dos últimos artículos - sobre Lola Cendales y Clotilde - tratan de profesoras cuya vida fue la expresión de la investigación en pedagogía. Tratan del pensamiento y de las practicas propias de ese campo del saber. Para concluir se puede decir que este número de la revista se hizo con el objetivo de contribuir al debate sobre la formación de los intelectuales y la preparación de los cuadros políticos y dirigentes de la educación en distintos países de América Latina. Resta la pregunta sobre las nuevas posibilidades de formación y de circulación internacional configuradas después de las crises económicas mundiales más recientes y de la emergencia de 
países como China, India, Brasil y Rusia en el escenario mundial.

\section{Agueda Bernardete Bittencourt $^{5}$}

\section{Referencias}

ALMEIDA, A. M. CANÊDO, L.B., GARCIA, A. BITTENCOURT, A. B., Circulação Internacional e Formação Intelectual das Elites Brasileiras. Campinas, SP: Editora da Unicamp, 2004.

BEIGEL, Fernanda, La Flacso chilena y la regionalización de las ciencias sociales en América Latina (19571973), Revista Mexicana de Sociologia 71 (2): 319-349.

BOURDIEU, P. Décrire et prescrire. Note sur les conditions de posibilité et les limites de l'eficacité politique, Actes de la Recherche en Science Sociales, 38, mai 1981, p. 69-73.

-----. Les conditions sociales de la circulation international des idées, Actes de la Recherche en Science Sociales, 145, 2002.

COOPER, F., T. Holt. \& R. Scott. Além de la escravidão: Investigaciones sobre raça, trabalho y cidadania en sociedades pos-emancipación. (Prefácio de Hebe Mattos), Rio de Janeiro: Civilización Brasileira, 2005. COOPER, Frederick, Le concept de mondialisation sert-il à quelque chose? Un point de vue d'historien,
Critique internationale $\mathrm{n}^{\circ} 10$ - janvier 2001, p. 101-124. CORBALÁN, A. Banco mundial. Intervención y disciplinamiento. El caso Argentino, enseñanzas para América Latina. Buenos Aires: Biblos, 2002.

DEZALAY, Yves, La mondialisation des guerres de palais, la estructuration du pouvoir d'état en Amérique Latine, entre notables du droit et "Chicago boys": Paris: Seuil, 2002.

Les courtiers de I'international, Actes de la Recherche en Sciences Sociales, 151-152, mars, 2004, p. 5-35.

HERRERA, M. C.; BITTENCOURT, A. B. Política, Intelectuales y espacio público en las sociedades contemporáneas, Bogotá: Cooperativa Editorial Magisterio, 2007.

KARADY, Viktor (1998), "La république des lettres des temps modernes: L'internationalisation des marchés universitaires occidentaux avant la Grande Guerre", Actes de la recherche en sciences sociales, no121-22 : 92-10.

Mobilité universitaire et circulation internationale des idées: le Brésil et la mondialisation des savoirs Cahiers de la recherche sur l'éducation et les savoirs, hors-série n²/juin 2009.

5 Profesora del Departamento de Educação, Conhecimento Linguagem e Arte/FE/Unicamp, investigadora del Grupo de Estudios sobre Instituição Escolar y Organizações Familiares - FOCUS.Correo: agueda@unicamp.br 